\title{
$\mathrm{LiF}$ 和 LiCl 对石墨电极电化学性能的影响
}

\author{
任䑣庄全超* 郝玉婉崔永丽 \\ (中国矿业大学 材料科学与工程学院锂离子电池实验室 徐州 221116)
}

\begin{abstract}
摘要 六氟磷酸锂是目前商品化锂离子电池中使用最广泛的电解质锂盐, $\mathrm{LiF}$ 和 $\mathrm{LiCl}$ 是除水和酸之外六氟磷酸锂产品 中最重要的杂质. 运用扫描电子显微镜 (SEM)、充放电、循环伏安法 $(\mathrm{CV})$ 以及电化学阻抗谱测试( $\mathrm{EIS}$ )等研究了 $\mathrm{LiF}$ 和 $\mathrm{LiCl}$ 对石墨电极电化学性能的影响. 充放电结果表明, 在 $1 \mathrm{~mol} / \mathrm{L} \mathrm{LiPF}$-EC:DEC:DMC 电解液中添加饱和的 LiF, 可以 显著提高石墨电极的充放电可逆容量并改善其循环性能, 而在 $1 \mathrm{~mol} / \mathrm{L} \mathrm{LiPF} 6$ - EC:DEC:DMC 电解液中添加饱和的 $\mathrm{LiCl}$, 虽也可提高石墨电极的首次充电容量, 但严重恶化石墨电极的充放电循环稳定性. CV 结果表明, 电解液中 $\mathrm{LiF} 、 \mathrm{LiCl}$ 的存在对 EC 的还原分解过程影响较小. 但 SEM 和 EIS 的结果指示, LiF、 LiCl 对石墨电极表面 SEI 膜的形成过程影响 较大. 在添加饱和 $\mathrm{LiF}$ 的电解液中石墨电极表面形成的 SEI 膜较薄且电阻较小, 进而提高了石墨电极的可逆循环容量及 改善了其循环稳定性; 但在饱和的 $\mathrm{LiCl}$ 电解液中石墨电极表面形成的 SEI 膜较厚且电阻较大, 严重恶化石墨电极的电 化学循环稳定性.
\end{abstract}

关键词＼cjkstart锂离子电池; 石墨电极; 六氟磷酸锂; $\mathrm{LiF} ; \mathrm{LiCl}$

\section{Influence of Electrochemical Performance of Lithium Ion Batteries with the Adding of $\mathrm{LiF}$ and $\mathrm{LiCl}$}

\author{
Ren, Tong Zhuang, Quanchao* Hao, Yuwan Cui, Yongli \\ (China University of Mining \& Technology, School of Materials Science \& Engineering of Lithium Ion Battery Lab, Xuzhou \\ 221116)
}

\begin{abstract}
In the past few decades, lithium hexafluorophosphate $\left(\mathrm{LiPF}_{6}\right)$ is the most widely employed ionic component in organic electrolyte solutions for commercial lithium ion battery, which is manufactured using $\mathrm{PCl}_{5}$, $\mathrm{LiF}$ and $\mathrm{HF}$ as raw materials via the HF solvent method in the large scale production, and then it commonly contains $\mathrm{LiF}$ and $\mathrm{LiCl}$ impurities besides water and acid. However, the influence of $\mathrm{LiF}$ and $\mathrm{LiCl}$ on the performance of lithium ion battery is still not clear. Thus, in this paper, the influence of $\mathrm{LiF}$ and $\mathrm{LiCl}$ on the electrochemical performance of graphite electrode was investigated using charge-discharge test and cyclic voltammetry (CV) combining with scanning electron microscope (SEM) and electrochemical impedance spectrum (EIS). Charge-discharge test results showed that the electrochemical performance of graphite electrode such as reversible capacity and cycling stability were significantly improved in $1 \mathrm{~mol} / \mathrm{L} \mathrm{LiPF}$-EC:DEC:DMC electrolyte with the saturation of $\mathrm{LiF}$. The initial charge capacity of graphite electrode in $1 \mathrm{~mol} / \mathrm{L} \mathrm{LiPF}_{6}-\mathrm{EC}: \mathrm{DEC}: \mathrm{DMC}$ electrolyte with the saturation of $\mathrm{LiF}$ is $331.0 \mathrm{mAh} / \mathrm{g}$, which is higher than that in $1 \mathrm{~mol} / \mathrm{L} \mathrm{LiPF}$-EC:DEC:DMC electrolyte $(307.9 \mathrm{mAh} / \mathrm{g})$. After 65 charge-discharge cycles, the charge capacity of graphite electrode in 1 mol/L LiPF $-E C: D E C: D M C$ electrolyte with the saturation of $\mathrm{LiF}$ is $340.1 \mathrm{mAh} / \mathrm{g}$, which is also higher than that in $1 \mathrm{~mol} / \mathrm{L} \mathrm{LiPF}_{6}-\mathrm{EC}: \mathrm{DEC}: \mathrm{DMC}$ electrolyte $(297.0$ $\mathrm{mAh} / \mathrm{g}$ ). However, although the first charging capacity of graphite electrode was enhanced in $1 \mathrm{~mol} / \mathrm{L} \mathrm{LiPF}-\mathrm{EC}_{\mathrm{DEC}} \mathrm{DMC}$ electrolyte with the saturation of $\mathrm{LiCl}$, the charge-discharge cycling stability was serious deteriorated. The initial charge capacity of graphite electrode in $1 \mathrm{~mol} / \mathrm{L} \mathrm{LiPF}_{6}$-EC:DEC:DMC electrolyte with the saturation of $\mathrm{LiCl}$ is $334.2 \mathrm{mAh} / \mathrm{g}$, yet after 65 charge-discharge cycles, the charge capacity of graphite electrode in $1 \mathrm{~mol} / \mathrm{L} \mathrm{LiPF}_{6}-\mathrm{EC}: \mathrm{DEC}: \mathrm{DMC}$ electrolyte with the saturation of $\mathrm{LiCl}$ is $251.2 \mathrm{mAh} / \mathrm{g}$. CV results showed that the influence of $\mathrm{LiF}$ and $\mathrm{LiCl}$ on the decomposition process of $\mathrm{EC}$ in electrolyte is small. SEM and EIS results stated that the SEI film which was formed on the graphite electrode is thinner and has a smaller resistance in $1 \mathrm{~mol} / \mathrm{L} \mathrm{LiPF}_{6}-\mathrm{EC}: \mathrm{DEC}: \mathrm{DMC}$ electrolyte with the saturation of $\mathrm{LiF}$ than that in $1 \mathrm{~mol} / \mathrm{L}$ $\mathrm{LiPF}_{6}$-EC:DEC:DMC electrolyte. Thus the reversible cycle capacity of graphite electrode was increased and its cycle stability was improved. Nevertheless the SEI film which was formed on the graphite electrode is thicker and its resistance is higher in $1 \mathrm{~mol} / \mathrm{L} \mathrm{LiPF}_{6}-\mathrm{EC}: \mathrm{DEC}: \mathrm{DMC}$ electrolyte with the saturation of $\mathrm{LiCl}$ than that in $1 \mathrm{~mol} / \mathrm{L} \mathrm{LiPF}_{6}$-EC:DEC:DMC electrolyte, which leads to the deterioration of electrochemical performance of graphite electrode.
\end{abstract}

Keywords lithium ion battery; graphite electrode; $\mathrm{LiPF}_{6} ; \mathrm{LiF} ; \mathrm{LiCl}$

\footnotetext{
* E-mail: zhuangquanchao@126.com

Received August 5, 2016; published October 13, 2016.
} 


\section{1 引言}

锂离子电池电解液主要由电解质锂盐、有机溶剂和 添加剂组成, 在过去的二十多年中, 六氟磷酸锂一直是 商品化锂离子电池电解液中使用最广泛的电解质锂盐, 可以预见未来六氟磷酸锂仍然会长期作为锂离子电池 电解液的主导电解质锂盐 ${ }^{[1]}$. 六氟磷酸锂的工业化大规 模生产主要采用氟化氢溶剂法 ${ }^{[2]}$, 反应式如下:

$$
\mathrm{LiF}+\mathrm{PF}_{5} \stackrel{\mathrm{HF}}{\longrightarrow} \mathrm{LiPF}_{6}
$$

由于五氟化磷与氟化锂都易溶于氟化氢中, 因此可 以在液相中发生均相反应, 使整个反应易于进行和控 制. 此外, 产品的结晶较容易分离, 易于实现工业化. (1)式中使用到的五氟化磷原料, 通常采用五氯化磷与 氟化氢反应制备 ${ }^{[3]}$ ，其反应式如下:

$$
\mathrm{PCl}_{5(\mathrm{~s})}+5 \mathrm{HF} \rightarrow \mathrm{PF}_{5(\mathrm{~g})}+5 \mathrm{HCl}_{(\mathrm{g})}
$$

由于缺乏市售的五氟化磷产品, (2)式所对应五氟化 磷的生产通常都是六氟磷酸锂生产线重要组成部分. 因 此, 六氟磷酸锂产品中除含有微量水和氟化氢等杂质 外, 必定还含有 $\mathrm{LiF}$ 和 $\mathrm{LiCl}$ 等杂质. 水和氟化氢等杂质 对锂离子电池性能的影响, 前人曾进行过深入的研 究 $^{[4 \sim 6]}$. 然而, 作为同样重要的杂质, $\mathrm{LiF}$ 和 $\mathrm{LiCl}$ 对锂离 子电池性能的影响, 却鲜见文献报道. 因此, 在本研究 工作中, 运用电化学阻抗谱并结合循环伏安、充放电测 试以及扫描电子显微镜等研究方法, 探讨了 $\mathrm{LiF}$ 和 $\mathrm{LiCl}$ 对石墨电极电化学性能的影响及其机制.

\section{2 实验部分}

实验在自制三电极玻璃电解池和两电极扣式电池 中完成, 金属锂片作为参比和辅助电极, 石墨电极由 90\%的石墨碳纤维(Petoca, Japan)、 $5 \%$ 的 PVDF-HFP (HS910, Elf2atochem, USA)粘合剂和 5\% 导电炭黑(上海 杉杉)组成, 涂覆活性材料量为 $1 \mathrm{mg} / \mathrm{cm}^{2}$. 空白电解液 为 $1 \mathrm{~mol} / \mathrm{L} \mathrm{LiPF}_{6}-\mathrm{EC}: \mathrm{DEC}: \mathrm{DMC}$ (体积比为 $1: 1: 1$ ) (张家 港国泰华荣化工新材料公司); $\mathrm{LiF}$ 和 $\mathrm{LiCl}$ 经 $80{ }^{\circ} \mathrm{C}$ 真空 干燥 $12 \mathrm{~h}$ 后, 直接使用. 充放电测试在 LANHE 蓝电电 池检测系统(武汉蓝电科技股份有限公司)上完成, 充放 电电压范围是 $0.005 \sim 3 \mathrm{~V}$, 充放电倍率为 $0.1 \mathrm{C}$, 充放电 采用 2032 扣式电池.

$\mathrm{CV}$ 和 EIS 测试均在电化学工作站(CHI660B, 上海 辰华仪器厂)上完成, 电极片规格为 $2 \mathrm{~cm} \times 2 \mathrm{~cm}$. 循环 伏安实验中, 电位扫描速度为 $1 \mathrm{mV} / \mathrm{s}$. 电化学阻抗实验 中频率的测试范围为 $10^{5} \sim 10^{-2} \mathrm{~Hz}$, 施加的交流信号振 幅为 $5 \mathrm{mV}$. 在进行阻抗测试前, 电极在极化电位平衡 1 h. 三电极体系装好后搁置 $2 \mathrm{~h}$ 再进行测试, 以保证电解 液对电极材料均匀湿润. 实验中获得的阻抗数据用 Zview 软件进行拟合. 电极形貌在 LEO 1530 场发射扫
描电子显微镜(FE-SEM, Oxford Instrument)上进行观察.

\section{3 结果与讨论}

\section{1 循环伏安研究结果}

如图 1 所示为石墨电极在未添加(空白电解液)和添 加饱和 $\mathrm{LiF}$ 与 $\mathrm{LiCl}$ 电解液中前 10 周的循环伏安扫描曲 线. 从图 1(a)可以看出, 石墨电极在空白电解液中, 首 次负向电位扫描过程中出现三个还原电流峰(峰 $\alpha$, 峰 $\beta$ 和峰 $\gamma$ ). 在随后的循环扫描过程中, 电极电位在 $1.0 \sim$ $0.4 \mathrm{~V}$ 之间的峰 $\alpha$ 和峰 $\beta$ 消失, 因此峰 $\alpha$ 和峰 $\beta$ 可归因于 溶剂分子 EC 的还原分解在石墨电极表面形成了 SEI 膜 的过程. 据 Naji 等 ${ }^{[7]}$ 的研究结果可知, 溶剂分子 EC 的还 原分解过程主要分为两个过程，如下所示:

第一步(双电子还原过程)

$$
\mathrm{EC}+2 \mathrm{e}^{-}+2 \mathrm{Li}^{+} \rightarrow \mathrm{LiCO}_{3}+\mathrm{CH}_{2}=\mathrm{CH}_{2}
$$

第二步(单电子还原过程)

$$
\begin{aligned}
& \mathrm{EC}+\mathrm{e}^{-}+\mathrm{Li}^{+} \rightarrow\left[\mathrm{Li}^{+} \mathrm{EC}^{-}\right] \\
& 2\left[\mathrm{Li}^{+} \mathrm{EC}^{-}\right] \rightarrow\left(-\mathrm{CH}_{2} \mathrm{OCO}_{2} \mathrm{Li}\right)_{2}+\mathrm{C}_{2} \mathrm{H}_{4} \\
& 2\left[\mathrm{Li}^{+} \mathrm{EC}^{-}\right] \rightarrow\left(-\mathrm{CH}_{2}-\mathrm{CH}_{2}-\mathrm{OCO}_{2} \mathrm{Li}\right)_{2}
\end{aligned}
$$

因此, 在 $0.75 \mathrm{~V}$ 左右出现的还原电流峰 $\alpha$ 可归因于 $\mathrm{EC}$ 还原分解生成碳酸锂 $\left(\mathrm{Li}_{2} \mathrm{CO}_{3}\right)$ 的过程, 在 $0.45 \mathrm{~V}$ 左右 生成的还原电流峰 $\beta$ 可归因于 $\mathrm{EC}$ 还原分解生成烷基碳 酸锂 $\left(\mathrm{ROCO}_{2} \mathrm{Li}, \mathrm{R}=-\mathrm{CH}_{2},-\mathrm{CH}_{2} \mathrm{CH}_{2} \ldots\right)$ 的过程. 另外在 0 $\mathrm{V}$ 附近出现的峰 $\gamma$ 与在正向扫描出现的电流峰构成的一 对氧化还原峰分别对应锂离子的嵌入/脱出过程.

从图 1(b)和 1(c)可以看出, 石墨电极在添加饱和 $\mathrm{LiF} 、 \mathrm{LiCl}$ 电解液中具有与空白电解液中类似的 $\mathrm{CV}$ 曲 线, 显示电解液中 $\mathrm{LiF} 、 \mathrm{LiCl}$ 的存在对 $\mathrm{EC}$ 的还原分解过 程影响较小. 与在空白电解液中对比, 石墨电极在添加 了饱和 $\mathrm{LiF}$ 的电解液中, 循环伏安曲线表现出较好的重 合性, 表明在添加饱和 $\mathrm{LiF}$ 的电解液中, 可改善石墨电 极的电化学性能. 然而, 与在空白电解液中对比, 石墨 电极在添加了饱和 $\mathrm{LiCl}$ 的电解液中, $\mathrm{CV}$ 曲线的重合性 较差且其首次循环过程中不可逆容量明显增大，表明在 添加有饱和 $\mathrm{LiCl}$ 的电解液中, 锂离子在石墨电极中嵌 入/脱出变得困难.

\section{2 充放电测试结果}

图 2 和 3 分别为石墨电极在空白电解液和添加了饱 和的 $\mathrm{LiF}$ 与 $\mathrm{LiCl}$ 电解液中的充放电 $(1 、 2 、 65$ 周)曲线与 循环性能曲线. 可以看出石墨电极在空白电解液中的首 周充放电容量分别为 $307.9 \mathrm{mAh} / \mathrm{g} 、 376.3 \mathrm{mAh} / \mathrm{g}$, 首周 库伦效率为 $81.8 \%$. 电解液在加入饱和 $\mathrm{LiF}$ 以后, 首周 

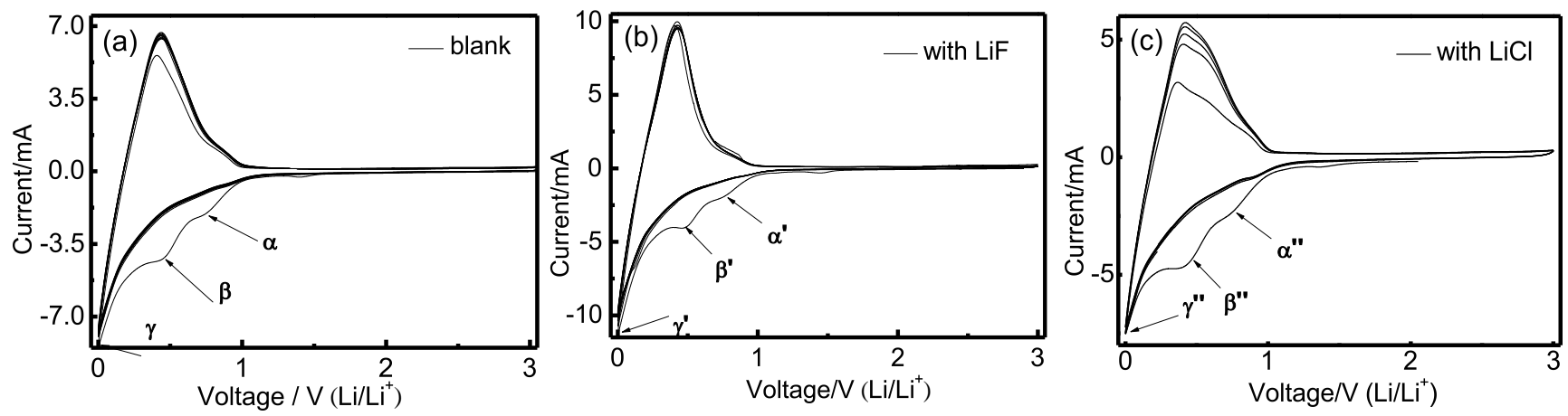

图 1 石墨电极在不同电解液体系下的循环伏安曲线. (a)空白, (b)添加 $\mathrm{LiF}$, (c)添加 $\mathrm{LiCl}$

Figure 1 Cyclic voltammetry curves of graphite electrode using the different electrolyte of system. (a) blank, (b) with $\mathrm{LiF}$, (c) with $\mathrm{LiCl}$
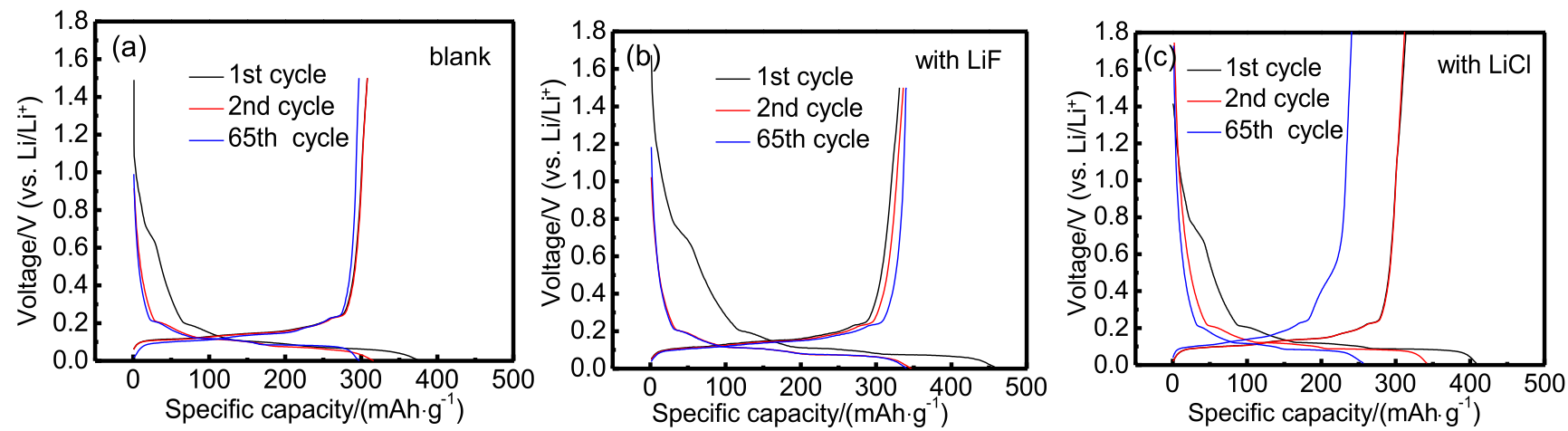

图 2 石墨电极在不同电解液体系下的充放电曲线. (a)空白, (b)添加 $\mathrm{LiF}$, (c)添加 $\mathrm{LiCl}$

Figure 2 Charge-discharge characteristics of graphite electrode using the electrolyte of (a) blank, (b) with LiF and (c) with $\mathrm{LiCl}$

充放电容量分别为 $331.0 \mathrm{mAh} / \mathrm{g} 、 461.8 \mathrm{mAh} / \mathrm{g}$, 首周库 伦效率为 $71.7 \%$. 电解液在加入饱和 $\mathrm{LiCl}$ 以后, 首周充 放电容量分别为 $334.2 \mathrm{mAh} / \mathrm{g} 、 408.5 \mathrm{mAh} / \mathrm{g}$, 首周库伦 效率为 $81.8 \%$. 表明 $\mathrm{LiF}$ 加入会在一定程度上降低石墨 电极的首周库伦效率, 但是 $\mathrm{LiF}$ 与 $\mathrm{LiCl}$ 的加入都显著提 高了石墨电极的首周充电容量. 石墨电极在空白电解液 中经历过 65 周充放电循环以后充电容量为 297.0 $\mathrm{mAh} / \mathrm{g}$, 其充电容量保持率约为 $96.5 \%$; 在加入饱和 $\mathrm{LiF}$ 的电解液中, 经历过 65 周循环后石墨电极的充电容量 为 $340.1 \mathrm{mAh} / \mathrm{g}$, 其充电容量不仅没有发生衰减且略微 增加. 在加入饱和 $\mathrm{LiCl}$ 的电解液中, 经历过 65 周循环 后石墨电极的充电容量为 $251.2 \mathrm{mAh} / \mathrm{g}$, 其充电容量保 持率为 $75.2 \%$, 表明在 $1 \mathrm{~mol} / \mathrm{L} \mathrm{LiPF}$-EC:DEC:DMC 电 解液中添加饱和的 $\mathrm{LiF}$, 可以显著提高石墨电极的充放 电可逆容量并改善其循环性能, 而在 $1 \mathrm{~mol} / \mathrm{L}$ $\mathrm{LiPF}_{6}-\mathrm{EC}: \mathrm{DEC}: \mathrm{DMC}$ 电解液中添加饱和的 $\mathrm{LiCl}$, 虽也可 提高石墨电极的首次充电容量, 但严重恶化石墨电极的 充放电循环稳定性. 由此可推知, 六氟磷酸锂中 $\mathrm{LiCl}$ 的 含量应当严格控制.

\subsection{SEM 研究结果}

图 4 是石墨电极在经历电化学扫描前后的 SEM 图. 可以看出, 所用的石墨碳纤维是棒状结构, 棒的表面光

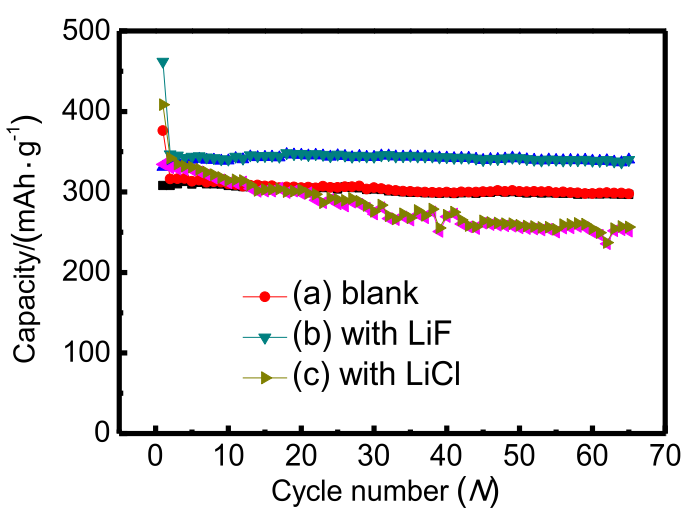

图 3 石墨电极在不同电解液体系下的循环性能曲线. (a)空白, (b)添 加 $\mathrm{LiF},(\mathrm{c})$ 添加 $\mathrm{LiCl}$

Figure 3 The cycle performance curve of graphite electrode using different electrolyte of system. (a) blank, (b) with $\mathrm{LiF}$, (c) with $\mathrm{LiCl}$

滑没有被 SEI 膜覆盖, 显示石墨电极表面在经历电化学 扫描前不存在初始 SEI 膜. 石墨电极在空白电解液中经 历 10 周电化学循环扫描后, 其表面明显被一层表面膜 (SEI 膜)所覆盖, 在低放大倍数下石墨碳纤维的棒状结 构已变得有些模糊不清. 石墨电极在添加饱和的 $\mathrm{LiF}$ 电 解液中经历 10 周电化学循环扫描后, 虽然其表面也同 样为一层 SEI 膜所覆盖, 但在低放大倍数下石墨碳纤维 
的棒状结构仍能很好地被观察到, 显示相比于在空白电 解液中, 石墨电极在添加饱和的 $\mathrm{LiF}$ 电解液中形成的表 面 SEI 膜要薄一些. 然而, 石墨电极在添加饱和的 $\mathrm{LiCl}$ 电解液中经历 10 周电化学循环扫描后, 已完全观察不 到石墨碳纤维的棒状结构, 表明石墨电极在添加饱和的 $\mathrm{LiCl}$ 电解液中形成表面 SEI 膜的厚度要远大于在空白电 解液和添加饱和的 $\mathrm{LiF}$ 电解液中形成的表面 SEI 膜厚度.
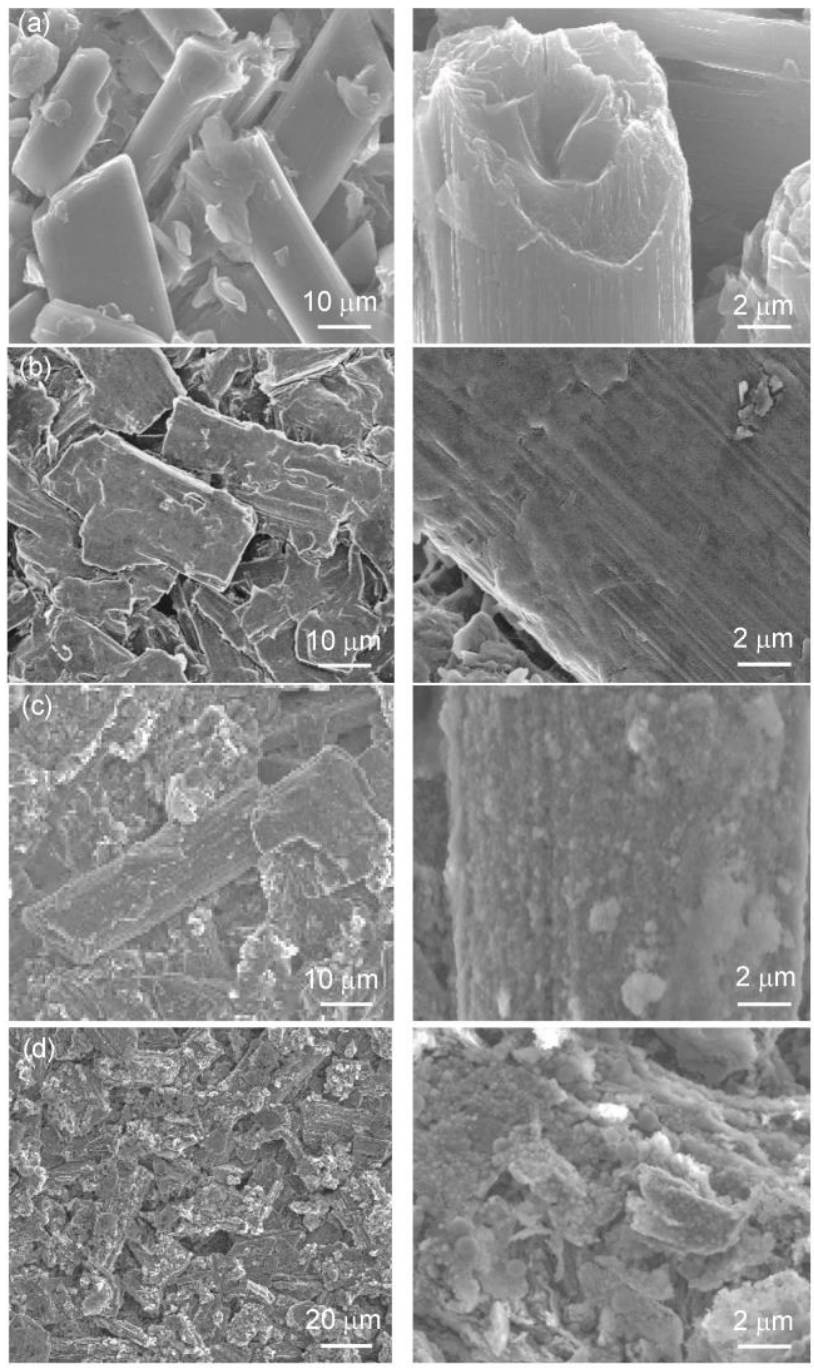

图 4 石墨电极(a)电化学测试之前, (b)空白, (c)添加 $\mathrm{LiF}$ 和(d)添加 $\mathrm{LiCl}$ 电化学测试之后的 SEM 图

Figure 4 SEM images of graphite electrode (a) before CV cycles, (b) blank, (c) with $\mathrm{LiF}$ and (d) with $\mathrm{LiCl}$ after $\mathrm{CV}$ cycles

\subsection{EIS 研究结果}

图 5 为石墨电极在空白和添加饱和的 $\mathrm{LiF}$ 与 $\mathrm{LiCl}$ 电解液中首次阴极极化过程的电化学阻抗谱. 可以看 出, 石墨电极在空白和添加饱和的 $\mathrm{LiF}$ 与 $\mathrm{LiCl}$ 电解液中 具有相似的 EIS 谱特征, 在开路电位 (3.0 V 附近)下, EIS 均由高频区的一个半圆(HFA)和低频区的圆弧(LFA)组 成. 考虑到石墨电极表面在进行电化学测试之前并不存 在初始的 SEI 膜(如 SEM 结果所示), 且在开路电位下也
不存在 SEI 膜的形成过程，因此此时的高频区的半圆应 归因于接触阻抗 ${ }^{[8 \sim 10]}$; 低频区域的圆弧则反映了此电位 下的石墨电极的阻滞电极特性 ${ }^{[11]}$. 随着电极极化电位 的降低, 电极极化电位在 $1.0 \mathrm{~V}$ 以上石墨电极在上述电 解液中的 EIS 谱特征基本保持不变, 表明当电极极化电 位高于 $1.0 \mathrm{~V}$ 时, 石墨电极表面不存在电解液组分还原 分解形成 SEI 膜和锂离子在石墨电极中嵌入的过程, 这 与 $\mathrm{CV}$ 的研究结果相一致.

随着电极极化电位的进一步降低, $1.0 \mathrm{~V}$ 时, 低频区 的圆弧演变成中频区域的一个半圆(MFA)和低频区域的 一条斜线. 此时 EIS 谱特征由三个部分组成，即高频区 半圆(HFA)、中频区半圆(MFA)和低频区的斜线(LFL) ${ }^{[12]}$. 根据 Aurbach 等 ${ }^{[13 \sim 17]}$ 的观点, HFA 应归因于锂离子通过 SEI 膜的迁移过程, MFA 则应归因于电荷传递过程, 而 LFL 则反映了锂离子在电极中的固态扩散的过程. 同时 考虑到, 在开路电位下, Nyquist 图的高频区域即存在一 个半圆, 因此 HFA 除与锂离子通过 SEI 膜的迁移过程 有关还应与接触阻抗有关, 由于接触阻抗随电极极化电 位的变化较小, HFA 的变化应主要与 SEI 膜有关. $1.0 \mathrm{~V}$ 以下, 随电极极化电位的降低, EIS 谱的特征基本保持 不变, 都由三部分组成.

为了进一步研究在电解液中添加 $\mathrm{LiF}$ 与 $\mathrm{LiCl}$ 对石墨 电极电化学性能的影响, 选取了图 6 所示的等效电路对 实验所获得的 EIS 谱图进行了拟合. 其中, $R_{\mathrm{s}}$ 代表欧姆 电阻, $R_{\mathrm{SEI}}$ 代表与 $\mathrm{SEI}$ 膜相关的电阻和接触阻抗, $R_{\mathrm{ct}}$ 代表 电荷传递电阻. SEI 膜电容 $C_{\mathrm{SEI}}$ 、双电层电容 $C_{\mathrm{dl}}$ 以及扩 散阻抗分别用恒相角元件 $(\mathrm{CPE}) Q_{\mathrm{SEI}} Q_{\mathrm{dl}}$ 和 $Q_{\mathrm{D}}$ 表示. CPE 能够表征复合电极的非理想行为(材料的多孔性, 电极 表面的粗䊁程度), 比较适合于模拟石墨负极 ${ }^{[18]}$.

图 7 为石墨电极在空白电解液和添加了饱和的 $\mathrm{LiF}$ 与 $\mathrm{LiCl}$ 电解液中首次阴极极化过程中的 $R_{\mathrm{SEI}} 、 R_{\mathrm{ct}}$ 随电 极极化电位的变化. 从图 7(a)可以看出, 在空白电解液 和添加了饱和的 $\mathrm{LiF}$ 与 $\mathrm{LiCl}$ 电解液中, $R_{\mathrm{SEI}}$ 随电极极化 电位的变化都表现出相近的变化趋势, 表明电解液中 $\mathrm{LiF}$ 与 $\mathrm{LiCl}$ 的存在对 $\mathrm{EC}$ 的还原分解过程影响较小, 这 与 $\mathrm{CV}$ 的研究结果基本一致. $R_{\mathrm{SEI}}$ 在电极极化电位从 1.0 $\mathrm{V}$ 降至 $0.7 \mathrm{~V}$ 的范围内存在一个上升的过程, 说明在这 个电位范围内石墨电极表面的 SEI 膜已经开始形成并随 着电极极化电位的降低而增厚, 这个现象应与电解液中 $\mathrm{EC}$ 还原分解有关 ${ }^{[7]}$, 这与 $\mathrm{CV}$ 的研究结果基本一致. $R_{\mathrm{SEI}}$ 在电极极化电位 $0.75 \mathrm{~V}$ 至 $0.3 \mathrm{~V}$ 范围内逐渐减小, 这种 现象可能与电解液单电子还原分解产物烷基碳酸锂与 电解液中痕量水反应生成 $\mathrm{Li}_{2} \mathrm{CO}_{3}{ }^{[19]}$ 的过程相关, 使得 石墨电极表面 SEI 膜组分中无机组分增加, 有利于对锂 离子传导. 在 $0.3 \sim 0.05 \mathrm{~V}$ 范围内, $R_{\mathrm{SEI}}$ 随电极极化电位 的降低略微增大, 这个现象可能是由于锂离子大量嵌入 石墨层间使石墨产生体积膨胀 ${ }^{[20]}$, 石墨电极表面的 SEI 膜不能适应这种体积变化而产生微小的破裂, SEI 膜破 

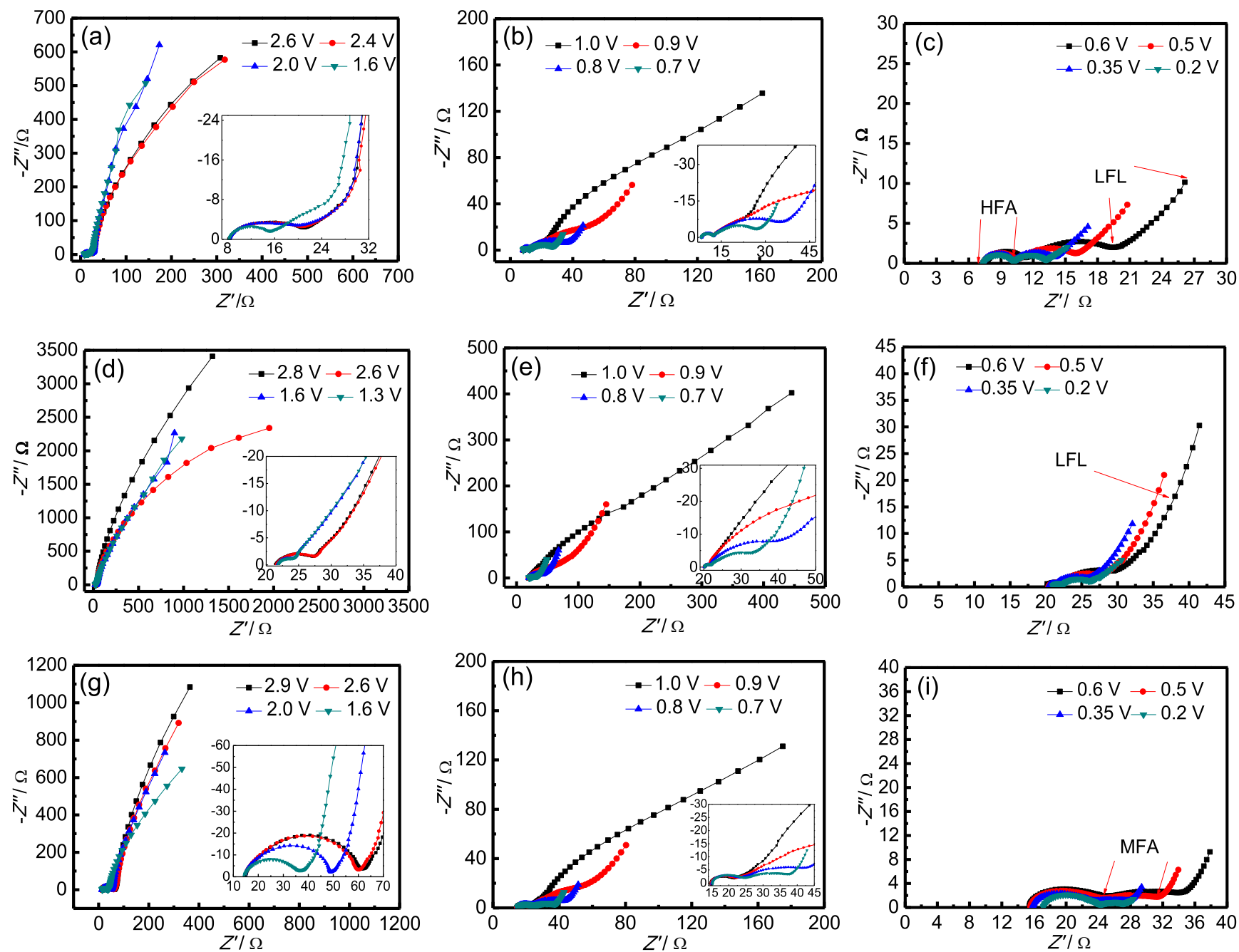

图 5 石墨电极在三种电解液中首次放电过程的电化学阻抗谱. (a) ( (c) 空白, (d) (f)添加 $\mathrm{LiF},(\mathrm{g}) \sim(\mathrm{i})$ 添加 $\mathrm{LiCl}$

Figure 5 Nyquist diagrams of the graphite electrode at various potentials from $3.0 \sim 0.2 \mathrm{~V}$ in different electrolytes in the first discharge process. (a) (c) blank, (d) (f) with $\mathrm{LiF},(\mathrm{g}) \sim(\mathrm{i})$ with $\mathrm{LiCl}$

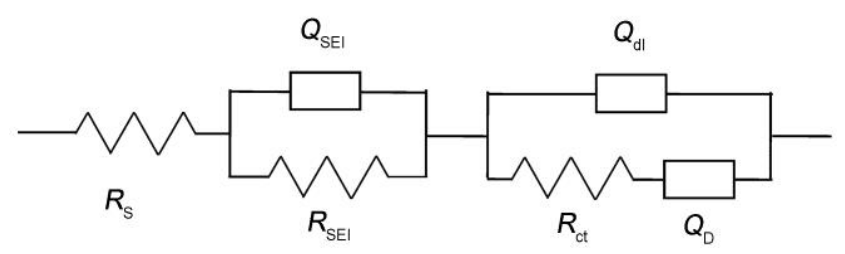

图 6 拟合 EIS 所需的等效电路

Figure 6 Equivalent circuit proposed for fitting impedance spectra

裂后会使石墨电极的新的活性面与电解液接触并与之 中的溶剂分子反应产生新的 SEI 膜, 起到 SEI 膜再修复, 这一破裂再修复的过程导致 $R_{\mathrm{SEI}}$ 增大 ${ }^{[21,22]}$. 此外, 相比 于在空白电解液中, 在添加了饱和 $\mathrm{LiF}$ 的电解液中 $R_{\mathrm{SEI}}$ 值明显减小, 说明在电解液中添加 $\mathrm{LiF}$ 可以使石墨表面 形成较薄的 SEI 膜, 从而提高了石墨电极的循环稳定性. 然而, 在添加了饱和 $\mathrm{LiCl}$ 添加剂的电解液中, $R_{\mathrm{SEI}}$ 的值 比在空白电解液中高很多, 表明在电解液中添加 $\mathrm{LiCl}$
将使石墨表面形成较厚的 SEI 膜, 从而导致电池的循环 稳定性降低, 这也与前面 SEM 分析结果相一致.

在空白电解液和添加了饱和的 $\mathrm{LiF}$ 与 $\mathrm{LiCl}$ 电解液 中, 与 $R_{\mathrm{SEI}}$ 类似, $R_{\mathrm{ct}}$ 也表现出相近的变化规律, 即随着 电压的降低 $R_{\mathrm{ct}}$ 逐渐下降. 但总体上, 在 $0.3 \mathrm{~V}$ 以下, 在 添加了饱和 $\mathrm{LiF}$ 电解液中的 $R_{\mathrm{ct}}$ 数值小于空白电解液中 的 $R_{\mathrm{ct}}$ 数值, 然而在添加饱和 $\mathrm{LiCl}$ 的电解液中的 $R_{\mathrm{ct}}$ 值大 于空白电解液中的 $R_{\mathrm{ct}}$ 数值, 显示在添加了饱和 $\mathrm{LiF}$ 的电 解液中锂离子嵌入/脱出更加容易, 进而提高了石墨电 极的循环性能, 而在添加了饱和 $\mathrm{LiCl}$ 电解液中正好相 反.

\section{4 结论}

运用扫描电镜、充放电、 $\mathrm{CV}$ 和 $\mathrm{EIS}$ 等在 $1 \mathrm{~mol} / \mathrm{L}$ $\mathrm{LiPF}_{6}-\mathrm{EC}: \mathrm{DEC}: \mathrm{DMC}$ 电解液中研究了 $\mathrm{LiF} 、 \mathrm{LiCl}$ 对石墨 电极电化学性能的影响. 研究结果表明, 在 $1 \mathrm{~mol} / \mathrm{L}$ 

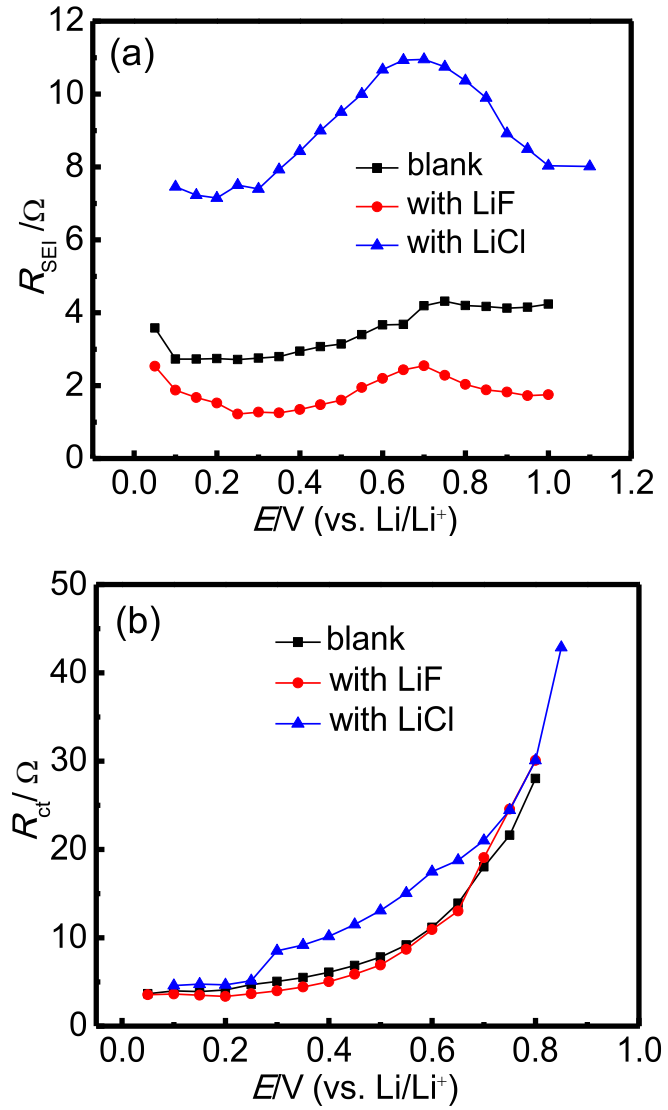

图 7 石墨电极首次阴极极化过程中的 $R_{\mathrm{SEI}}$ 和 $R_{\mathrm{ct}}$ 随电极极化电位的变 化

Figure 7 Variations of (a) $R_{\mathrm{SEI}}$ and (b) $R_{\mathrm{ct}}$ with the decrease of electrode polarization potential

$\mathrm{LiPF}_{6}-\mathrm{EC}: \mathrm{DEC}: \mathrm{DMC}$ 电解液中添加饱和的 $\mathrm{LiF} 、 \mathrm{LiCl}$ 对 EC 的还原分解过程影响较小, 但会影响 SEI 膜的成膜 过程. 相比于在空白电解液中, 在添加饱和 $\mathrm{LiF}$ 的电解 液中石墨电极表面形成的 SEI 膜较薄且内阻较小, 进而 提高了石墨电极的可逆循环容量并改善了其循环稳定 性; 然而, 在饱和的 $\mathrm{LiCl}$ 电解液中石墨电极表面形成的
SEI 膜较厚且内阻较大, 严重恶化石墨电极的电化学循 环稳定性. 因此, 在六氟磷酸锂的生产中, 产品中 $\mathrm{LiCl}$ (或 $\mathrm{Cl}^{-}$) 的残留量必须严格控制.

\section{References}

[1] Xu, K. Chem. Rev. 2004, 104, 4303.

[2] Zhuang, Q.-C.; Wu, S.; Liu, W.-Y.; Lu, Z.-D. Chin. Batt. Ind. 2005 , 10,169 (in Chinese). (庄全超, 武山, 刘文元, 陆兆达, 电池工业, 2005, 10, 169.)

[3] Li, J.; Tian, L.-L.; Zhao, F.-L.; Zhuang, Q.-C. Appl. Chem. Ind. 2011, 40, 524 (in Chinese). (李佳，田雷雷，赵封林，庄全超，应用 化工, 2011, 40, 524.)

[4] Aurbach, D.; Markovsky, B.; Shechter, A.; Ein-Eli, Y. J. Electrochem. Soc. 1996, 143, 3809.

[5] Aurbach, D.; Weissman, I.; Zaban, A.; Dan, P. Electrochim. Acta 1999, 45,1135

[6] Aurbach, D.; Schechter, A. Electrochim. Acta 2001, 46, 2395.

[7] Naji, A.; Ghanbaja, J.; Humbert, B.; Willmann, P.; Billaud, D. $J$. Power Sources 1996, 63, 33.

[8] Holzapfel, M.; Martinent, A.; Alloin, F.; Le Gorrec, B.; Yazami, R.; Montella, C. J. Electroanal. Chem. 2003, 546, 41.

[9] Du, L.-L.; Zhuang, Q.-C.; Wei, T.; Shi, Y.-L.; Qiang, Y.-H.; Sun, S.-G. Acta Chim. Sinica 2011, 69, 2641 (in Chinese). (杜莉莉, 庄全 超, 魏涛, 史月丽, 强颖怀, 孙世刚, 化学学报, 2011, 69, 2641.)

[10] Wei, T.; Zhuang, Q.-C.; Wu, C.; Cui, Y.-L.; Fang, L.; Sun, S.-G. Acta Chim. Sinica 2010, 68, 1481 (in Chinese). (魏涛, 庄全超, 吴超, 崔永丽，方亮，孙世刚，化学学报，2010，68, 1481.)

[11] Chang, Y.-C.; Sohn, H.-J. J. Electrochem. Soc. 2000, 147, 50.

[12] Zhuang, Q.-C.; Chen, Z.-F.; Dong, Q.-F.; Jiang, Y.-X.; Zhou, Z.-Y.; Sun, S.-G. Chem. J. Chin. Univ. 2005, 26, 2073 (in Chinese). (庄全 超, 陈作锋, 董全峰, 姜艳霞, 周志有, 孙世刚, 高等学校化学学 报, 2005, 26, 2073.)

[13] Levi, M.-D.; Aurbach, D. J. Phys. Chem. B 1997, 101, 4630.

[14] Levi, M.-D.; Aurbach, D. J. Power Sources 2005, 146, 727.

[15] Deng, X.; Xie, K.; Li, L.; Zhou, W.; Sunarso, J.; Shao, Z. Carbon 2016, 107, 67.

[16] Deng, X.; Zhao, B.; Zhu, L.; Shao, Z.-P. Carbon 2015, 93, 48

[17] Zhang, S.-S.; Xu, K.; Jow, T.-R. Electrochim. Acta 2006, 51, 1636.

[18] Zhang, S.; Shi, P. Electrochim. Acta 2004, 49, 1475.

[19] Xu, S.-D.; Zhuang, Q.-C.; Tian, L.-L.; Qin, Y.-P.; Fang, L.; Sun, S.-G. J. Phys. Chem. C 2011, 115, 9210.

[20] Zhuang, Q.-C.; Wei, T.; Wei, G.-Z.; Dong, Q.-F.; Sun, S.-G. Acto Chim. Sinica 2009, 67, 2184 (in Chinese). (庄全超, 魏涛, 魏国祯, 董全峰, 孙世刚, 化学学报, 2009, 67, 2184.)

[21] Wang, C.-S.; Kakwan, I.; John Appleby, A.; Little, F.-E. J. Electroanal. Chem. 2000, 489, 55.

[22] Wang, C.-S.; Appleby, A.-J.; Little, F.-E. J. Electroanal. Chem. 2001, 497, 33.

(Cheng, B.) 